\title{
Size-Dependent Phenomena in Angle-Resolved Measurements of Submicron Sn Particle Scattering from a Molybdenum Surface
}

Morgan E.C. Miller ${ }^{1}$, Michelle Mezher2, Silvia De Dea ${ }^{3}$, Robert E. Continetti2,"*

${ }^{1}$ Department of Nanoengineering, University of California, San Diego, La Jolla, California, USA 92093-0340

${ }^{2}$ Department of Chemistry and Biochemistry, University of California, San Diego, La Jolla, California, USA 92093-0340

${ }^{3}$ ASML US, 17082 Thornmint Ct.,San Diego, California, USA 92127

\section{Supplementary Information}

\section{Characterization of Tin Particles}

Tin particles collected directly from the AIS beamline were analyzed to determine the extent of oxidation within the particles. The samples were produced by collecting slightly off-axis tin particles deposited on an image charge detector aperture located directly before the electrostatic trap. The particles were collected using small strips of carbon tape applied to the tin particle residue. This sample of beamline particles was sent to EAG laboratories for analysis of composition and identification of the oxide layer. Collections of particles were identified, and cut into planes to allow annular dark-field (HAADF) imaging of particle cross sections with a scanning transmission electron

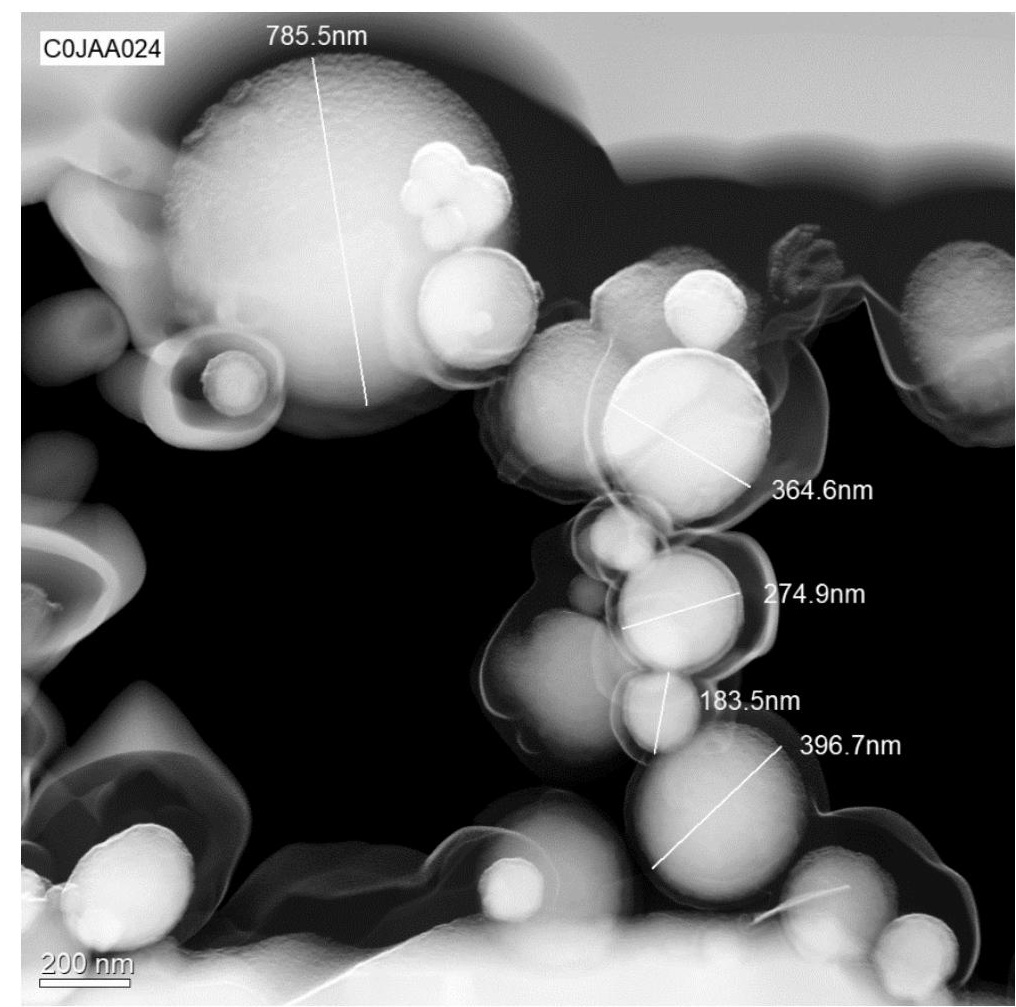

Figure S1: HAADF STEM imaging of individual tin particles collected from the AIS beamline. Particle sizes were added by the STEM operator as part of the analysis. A variety of particle sizes were collected within the residue sampled, allowing comparison of particle shape across different particle diameters. 
microscope (STEM). HAADF detection was desired to allow identification of the particle oxide layer. HAADF STEM imaging of individual particles is shown in Figure $\mathbf{S 1}$ with identification lines of particle size added.

Figure S2 shows two particles analyzed for oxide layer thickness. The two different sizes both contain a similarly thick $\sim 10 \mathrm{~nm}$ oxide layer that was found to be consistent across the particles sizes measured. To confirm the composition of the layer, energy-dispersive X-ray spectroscopy (EDS) analysis was performed on the particles used in the STEM. A color map of the key elements identified in this analysis is shown for a few particles in Figure S3. The oxide layer is clearly visible in the oxygen map for each particle and is confirmation of the analysis presented in Figure $\mathbf{S 2}$.

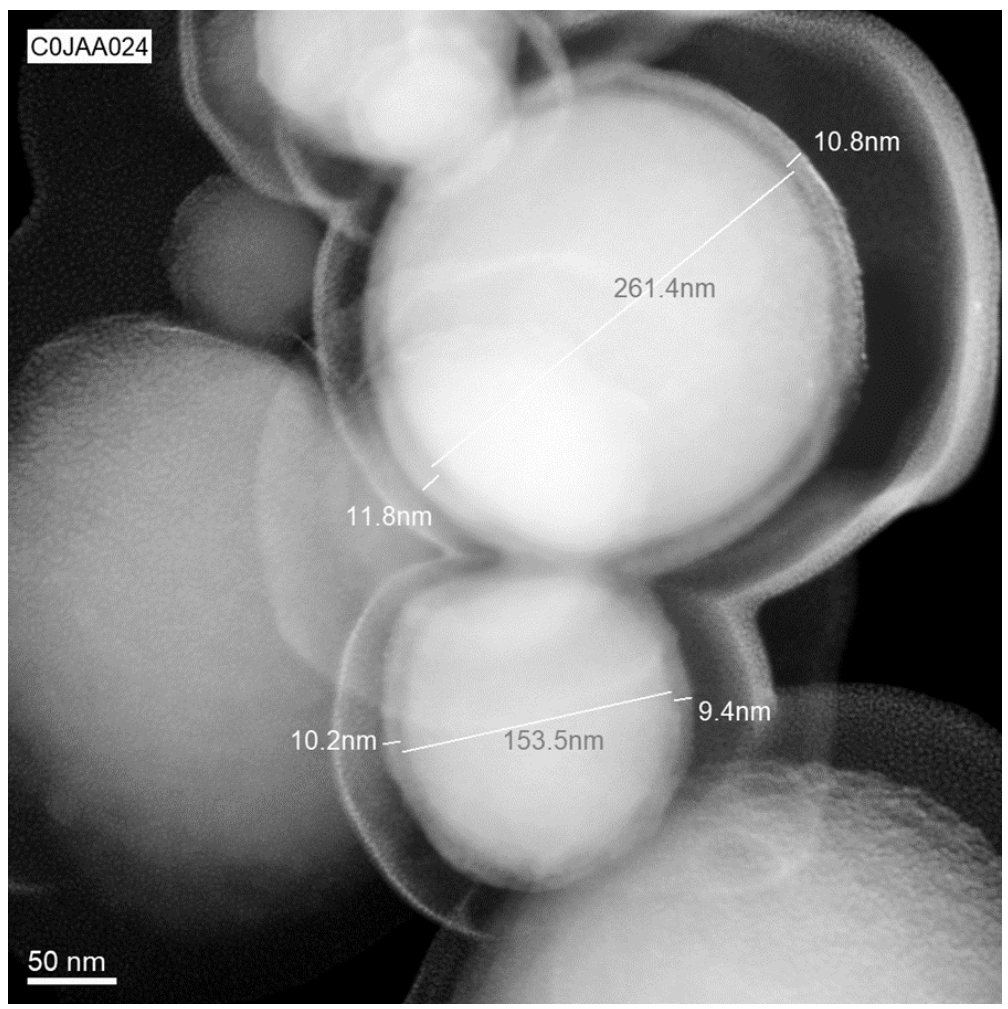

Figure S2: HAADF STEM imaging of individual tin particles for oxide layer thickness. Thickness of the oxide layer and particle diameter are annotated as shown. The average thickness for the oxide layer on particles analyzed was $\sim 10 \mathrm{~nm}$. 

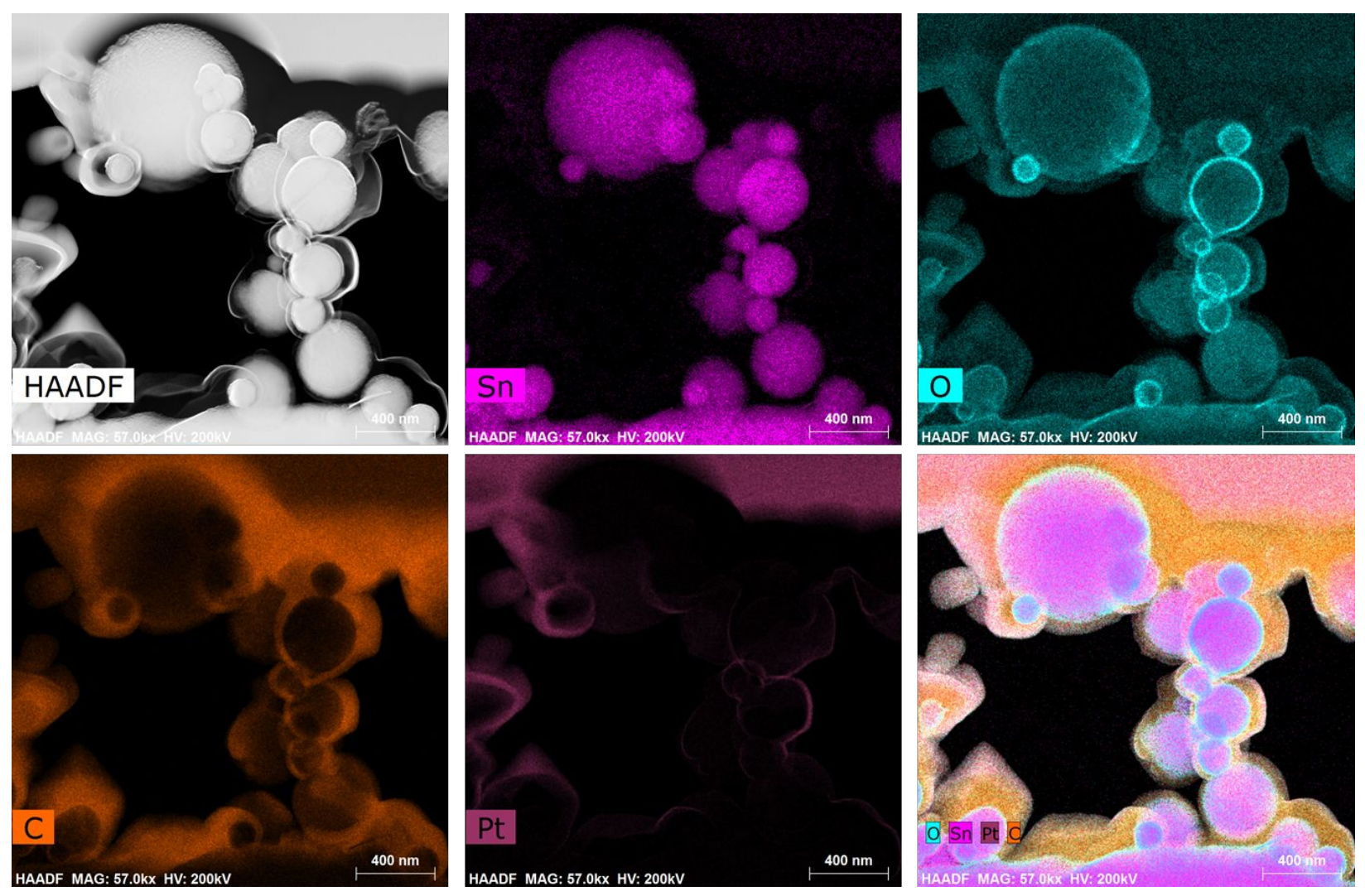

Figure S3: STEM EDS color mapping of major elements present in analysis with original HAADF STEM image. Platinum was used to coat the sample before analysis. Carbon is present from the carbon tape used in sample collection.

\section{Discussion of Fit Sensitivity to Physical Parameters}

As noted in the main text, the model used to fit the experimental CoR information does not perfectly match the data, limiting the ability to derive quantitative information from the data. These fits are made with many simplifications including: treating the material properties as static, treating the energetic loss as only a result of material deformation, and treating the initial particle as a homogenous perfect sphere. In addition to not being static properties the various parameters used to fit the simple model have different orders of impact on the curve itself. By varying the different parameters derived from the fit, the sensitivity of the final fit on each parameter can be demonstrated as done for $150 \mathrm{~nm}$ tin in Figure S4. 

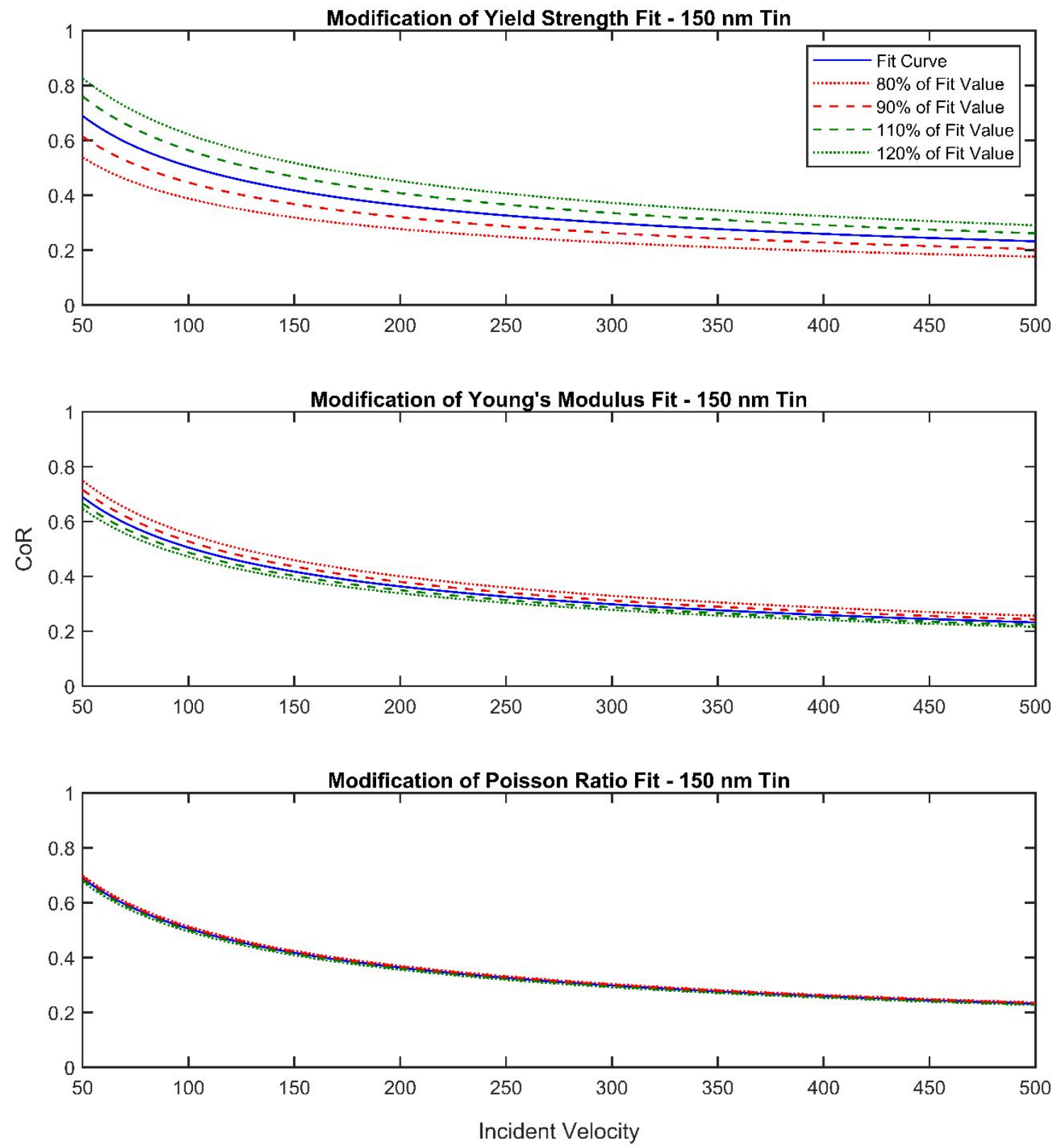

Figure S4: Curve fitting performed for the $150 \mathrm{~nm}$ tin CoR data. The initial fit (as presented in the main) is shown in blue in all three frames. Modification of one fit parameter (post-fit) is done for individually in each frame. Artificially increasing the parameter is show in green, while decreasing the parameter is shown in red.

The yield strength has the most significant factor in affecting the relative fit of the curve (specifically, its relative starting and ending CoR values), while both Young's modulus and Poisson ratio are significantly less impactful. This is the desired output of 
the model as it is assumed that yield strength is the property most likely to affect the fits and that Young's modulus and Poisson ratio are most likely to not change significantly form their bulk values (further justification of this can be found in the main text). However, due to the relative insensitivity of the fit to Young's modulus and Poisson ratio their true values for the tin particles used here cannot be derived from this fitting procedure. It is the intent of this fitting not to derive any realistic values for the tin materials used here, but rather under understand what parameters play the largest role in the size dependence seen in the experimental data. It is beyond the scope of this analysis to attempt to effectively quantize the error associated with this fitting procedure, particularly due to the significant assumptions made when using the analysis presented, as the error caused by these assumptions outweighs any fitting-based inaccuracy. 